\title{
Characteristics Analysis and Modelling of a Pneumatic Underwater Launching System
}

\author{
Bin $\mathrm{Li}^{1}$, Song Guo ${ }^{1}$, Wei Li ${ }^{1}$, Deman Zhang ${ }^{1}$, Nei Wang ${ }^{2}$ \\ ${ }^{1}$ Wuhan Second Ship Design and Research Institute, Wuhan, China \\ ${ }^{2}$ Wuhan Maritime Communications Research Institute, Wuhan, China
}

\begin{abstract}
Comprehensive characteristics of a pneumatic underwater launching system were analyzed and the simulation was carried out by simulink. The components of the pneumatic underwater launching system were introduced, and the theoretical calculation formula for the system was derived. A rated pressure of $3.5 \mathrm{MPa}$ and $5 \mathrm{MPa}$ was offered in the numerical work. Analyses in different piston height show good behaviors: Proper increase of piston-initial accumulator pressure is beneficial to reduce hydrodynamic noise, choose the appropriate pressure of accumulator. The hydrodynamic noise of the system can be significantly reduced by optimizing the structure of the double-acting cylinder, increasing the height of the piston and improving the structure of the piston.
\end{abstract}

\section{Introduction}

Pneumatic underwater launching system is a special power conversion device which uses compressed air as power source and seawater as working medium to realize non-rigid energy transmission. The system has many unfavourable factors, such as large structure volume and excessive hydrodynamic force produced during launching process. Therefore, it is necessary to optimize the design of the system to improve its overall performance.

A complete mathematical model of launching system is established, and the Simulink simulation toll is used to realize the whole system. In the process of simulation, the dynamic effects of different factors on the launching process can be observed conveniently and quickly. It provides a certain theoretical basis and an effective analytical tool for the optimization design of the system.

The composition and working principle of pneumatic launching system are shown in Fig 1. The system consists of high pressure accumulator, water chamber, canister launcher, double acting cylinder and control valve. When launching a weapon, the high-pressure air in the high pressure accumulator through the launching control valve to push the piston of double acting cylinder, which compresses the seawater through the water tank into the canister launcher, and the direction of pressure is as shown in the chart.

Mathematic model of pneumatic underwater launching system was established, and then simulation work was carried out. The results were pretty suggestive on optimal design and improvement of the pneumatic underwater launching system.

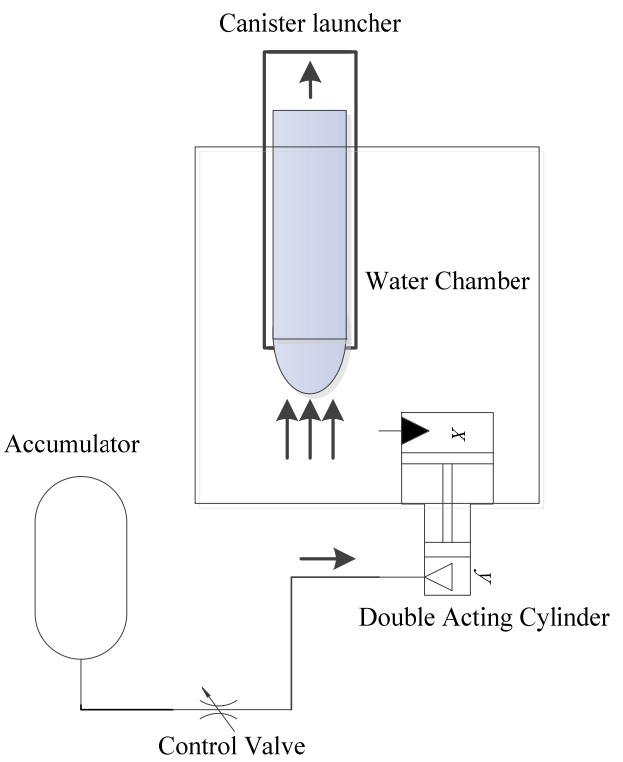

Fig 1 Cross-section of the dynamic flow balance valve

\section{Mathematic models}

The following assumptions were made to simplify the mathematic model.

1. According to the thermodynamic model and continuity equation of air, Transient pressure of cylinder gas

\footnotetext{
a Corresponding author: libinzju@sina.com, guosongjie555@163.com,93320399@qq.com, lin1985101@sina.com, neilwong2011@163.com
} 


$$
\begin{array}{r}
\dot{p}_{s}=-k \frac{p_{s}}{\rho} \dot{\rho} \\
\dot{T}_{s}=-(k-1) \frac{T_{s}}{\rho} \dot{\rho}
\end{array}
$$

- $p_{s}$ was pressure of accumulator.

- $k$ was adiabatic exponent.

- $\rho$ was gas density of accumulator.

- $T_{s}$ was gas temperature of accumulator.

2. Assuming that the pressure is uniform in each control body and the pressure flow equation in the double acting cylinder, water chamber and canister launcher can be obtained by using the fluid elasticity theory and continuity equation.

$$
\dot{p}_{w}=\left[-q_{s}+\left(S_{1}-S_{2}\right) \dot{x_{p}}\right] \frac{E}{V_{w}}
$$

- $p_{w}$ was pressure of cylinder.

- $x_{p}$ was piston displacement of cylinder.

- $\rho$ was gas density of accumulator.

- $E$ was the volume elastic modulus of water.

- $V_{w}$ was the valid volume of cylinder.

3. Pressure change of canister launcher

$$
\dot{p_{c}}=\left(q_{t i}-q_{t o}-\dot{V}_{0}\right) \frac{E}{V_{0}}
$$

4. The flow of water from the water chamber into the canister launcher

$$
q_{t i}=C \cdot S \sqrt{\frac{2}{\rho_{w}}\left(p_{w}-p_{c}\right)}
$$

- $C$ was flow characteristic coefficient.

- $S$ was the area of annular hole.

5. Pressure change of water chamber

$$
\dot{p}_{w}=\left(q_{w i}-q_{w o}\right) \frac{E}{V_{w}}
$$

6. The forces on the weapon are: gravity $G$, buoyancy $F$, head on resistance $F_{x}$, tube wall friction $F_{g}$, seawater thrust and external seawater static pressure. In the course of weapon movement, the volume left behind is replenished by seawater of the canister launcher. The motion equation of weapon is

$$
\dot{v}_{t}=\frac{1}{m}\left[\left(p_{w 2}-p_{w 1}\right) S-f_{g}-F_{x}-S \rho v_{t}^{2}\right]
$$

\section{Analysis}

Simulink was adopted for the mathematic characteristics simulation (as seen in Fig 2). The simulation is designed by modularization and consists of five submodules to simulate each component of the pneumatic underwater launching system. The basic module is implemented, so that the dynamic characteristics of the system can be observed and compared conveniently from each by the scope, thus improving the efficiency of system analysis.
The main parameters of the pneumatic underwater launching system are shown in Table 1.

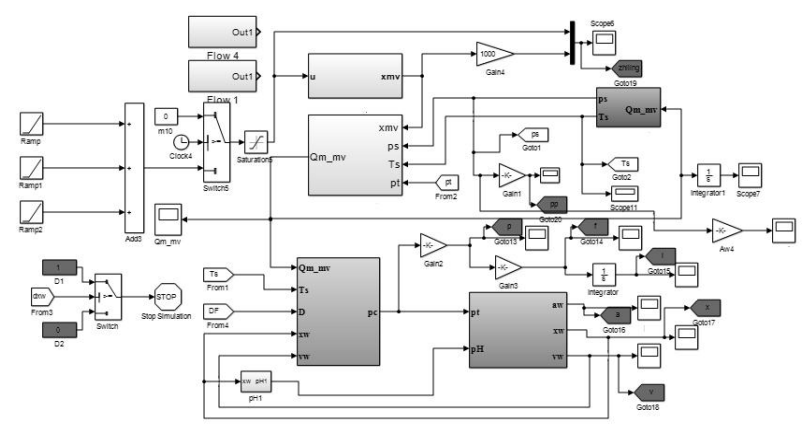

Fig 2 Diagrammatic of simulink simulation

Table 1 Simulation parameter setting list

\begin{tabular}{|c|c|}
\hline Variables & Value \\
\hline$V_{s}$ & $4 \mathrm{~L}$ \\
\hline$L$ & $1.225 \mathrm{~m}$ \\
\hline$p_{s}$ & $5 \times 10^{6} \mathrm{~Pa}$ \\
\hline$D$ & $20 \mathrm{~mm}$ \\
\hline$V_{0}$ & $10 \mathrm{~L}$ \\
\hline
\end{tabular}

\subsection{Influences by different height of piston- initial accumulator pressure at $5 \mathrm{MPa}$}

A various height difference of piston from $0.25 \mathrm{~m}$ to $0.75 \mathrm{~m}$ was given as the original input, Fig $3 \sim$ Fig 10 were different performances when simulation conditions changed.

In the process of system optimization, the structural constraints and the different effects of the stability and stiffness of the double acting cylinder on the piston are taken into account, $0.25 \mathrm{~m}, 0.5 \mathrm{~m}$ and $0.75 \mathrm{~m}$ are recommended to be set among $0.25 \mathrm{~m} \sim 0.75 \mathrm{~m}$.

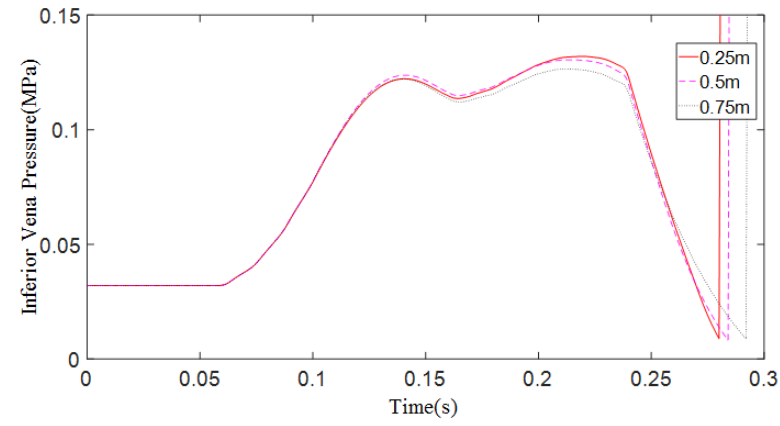

Fig 3 Change of inferior vena pressure in different time

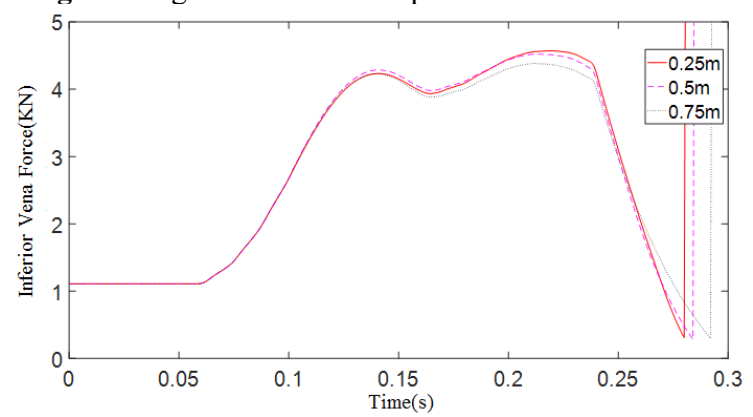

Fig 4 Change of inferior vena force in different time 


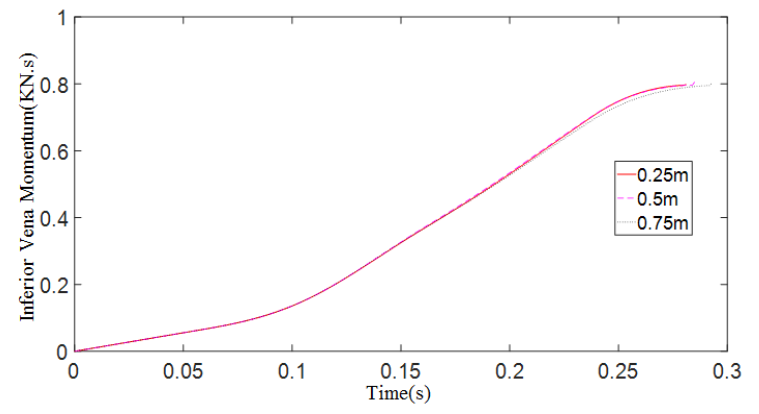

Fig 5 Change of inferior vena momentum in different time

It can be seen from Fig 3 to Fig 5 that with the increase of piston height, the change trend of the inferior vena force on the bottom of the piston is the same, and the time required is gradually increasing. This is for the piston when the height is small, the fixed part of the mass is small, and the acceleration is large, under the same travel conditions the shorter the motion time. The momentum is determined by the inferior vena force and the acting time. The total size depends on the curve of the inferior vena force changing with time and the size of the envelope area of the transverse axis. From the curve of the inferior vena force with time, it can be seen that the product of the three sets of height is not different, and the momentum ratio of the three sets of height is close. During the whole course of motion, the three groups of high piston were subjected to the action of high gas at the bottom force not more than $4.6 \mathrm{kN}$, and the pressure of lower cavity was not more than $0.14 \mathrm{MPa}$.

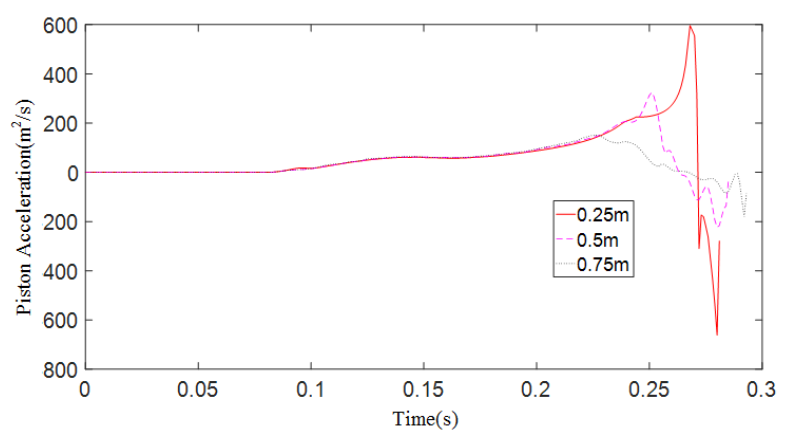

Fig 6 Change of piston acceleration in different time

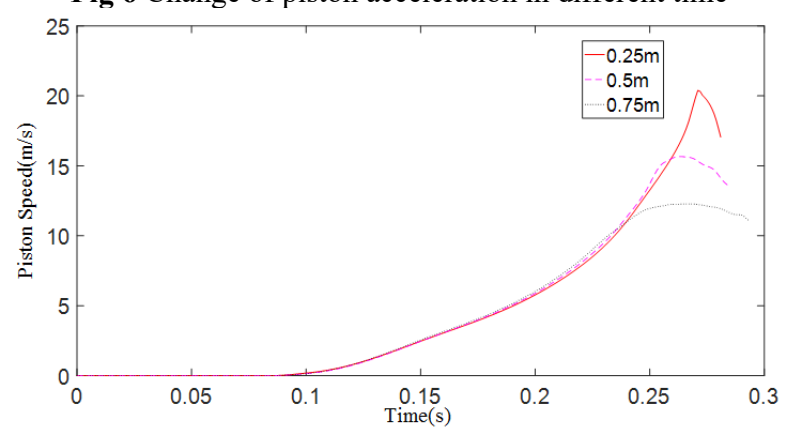

Fig 7 Change of piston speed in different time

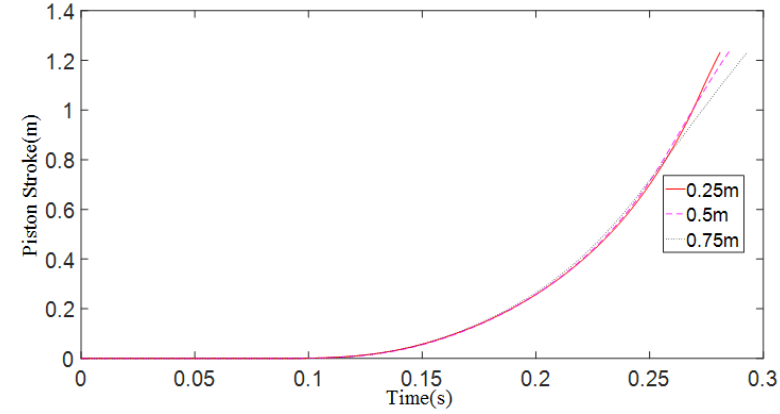

Fig 8 Change of piston stroke in different time

From Fig 6 to Fig 8, it can be found that with the increase of the height of the piston, the acceleration of the piston motion becomes smaller and smaller, and the time required for the same stroke is getting longer and the speed is getting smaller and smaller. This is because the smaller the height of the piston, the greater the mass of the water in the chamber before the outlet, and the more the mass is reduced after the exit, so the acceleration is greater. During the whole course of motion, the final velocity of the three sets of height piston is about $10 \mathrm{~m} / \mathrm{s}$ to $20 \mathrm{~m} / \mathrm{s}$, and the maximum acceleration corresponding to the $0.5 \mathrm{~m}$ piston is about $30 \mathrm{~g}$, the maximum acceleration corresponding to the $0.75 \mathrm{~m}$ piston is about $20 \mathrm{~g}$, and the acceleration corresponding to the $0.25 \mathrm{~m}$ piston is slightly larger.

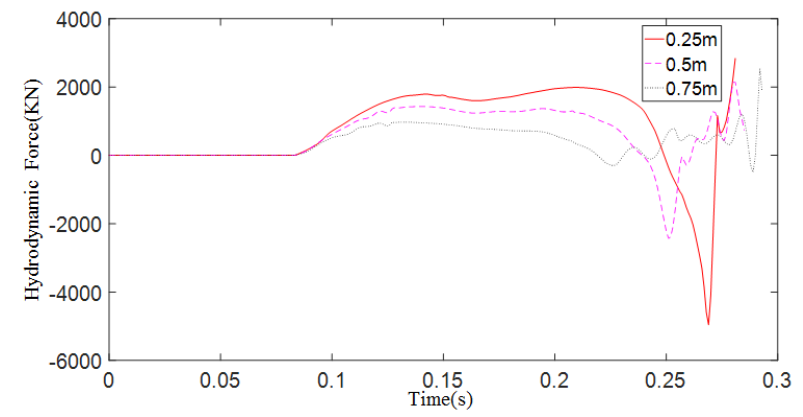

Fig 9 Change of hydrodynamic force in different time

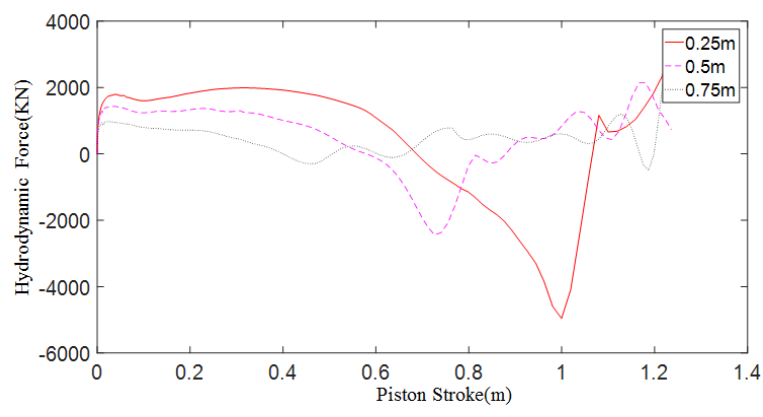

Fig 10 Change of hydrodynamic force in different piston stroke

As seen in Fig 9 and Fig 10, with the increase of the height of the piston, the hydrodynamic force as a whole shows a decreasing trend, and the bottom force does not change much and there is no obvious law of change. This is because the greater the height of the piston, the smaller the velocity, and the positive correlation between the hydrodynamic force and the square of the velocity, so the hydrodynamic force as a whole is decreasing gradually. As seen, curve at $0.75 \mathrm{~m}$ has good performance in hydrodynamic force. 


\subsection{Influences by different height of piston- initial accumulator pressure at $3.5 \mathrm{MPa}$}

The initial pressure of the accumulator changed from $5 \mathrm{MPa}$ to $3.5 \mathrm{MPa}$, and the opening rule of the control valve was changed to keep the other conditions unchanged. Using the same method, the simulation results are shown in from Fig 11 to Fig 18.

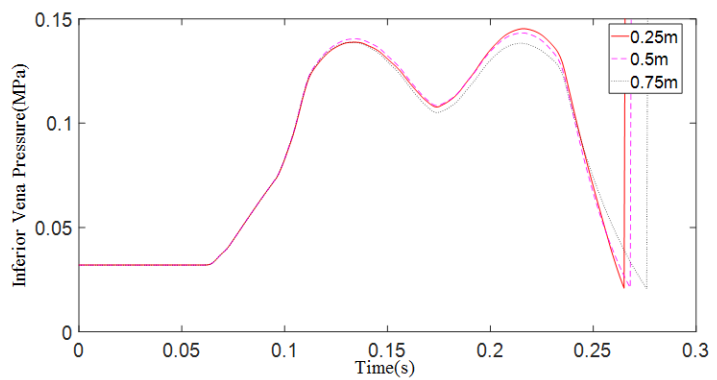

Fig 11 Change of inferior vena pressure in different time

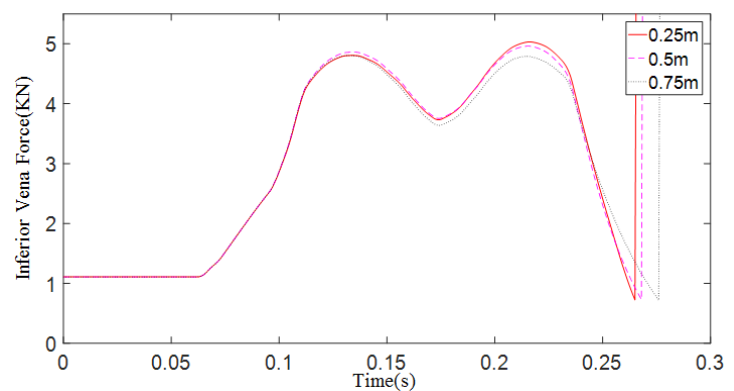

Fig 12 Change of inferior vena force in different time

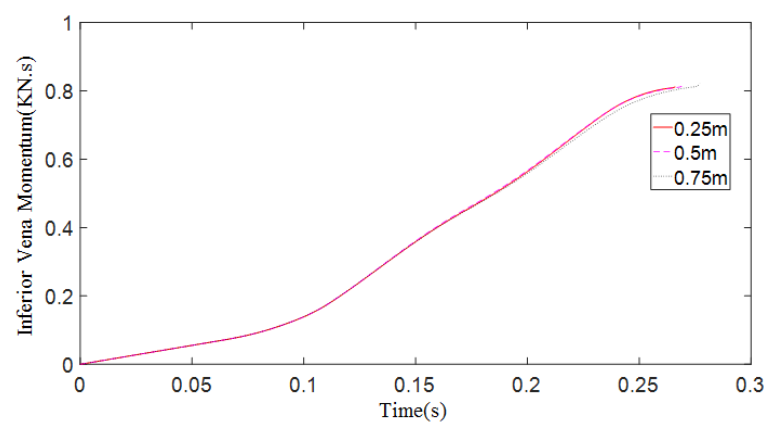

Fig 13 Change of inferior vena momentum in different time

It can be seen from Fig 11 to Fig 13 that with the increase of piston height, the change trend of the inferior vena force on the bottom of the piston is the same, and the time required is gradually increasing. This is for the piston when the height is small, the fixed part of the mass is small, and the acceleration is large, under the same travel conditions the shorter the motion time. The momentum is determined by the inferior vena force and the acting time. The total size depends on the curve of the inferior vena force changing with time and the size of the envelope area of the transverse axis. From the curve of the inferior vena force with time, it can be seen that the product of the three sets of height is not different, and the momentum ratio of the three sets of height is close. During the whole course of motion, the three groups of high piston were subjected to the action of high gas at the bottom force not more than $5 \mathrm{kN}$, and the inferior vena monentum was not more than $0.82 \mathrm{KN} \cdot \mathrm{m}$.

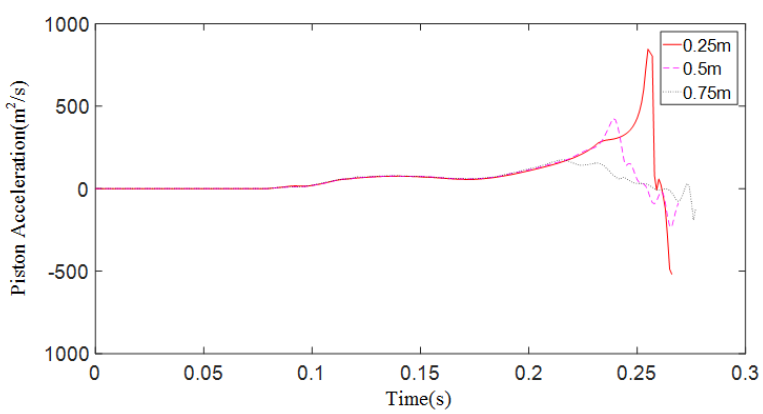

Fig 14 Change of piston acceleration in different time

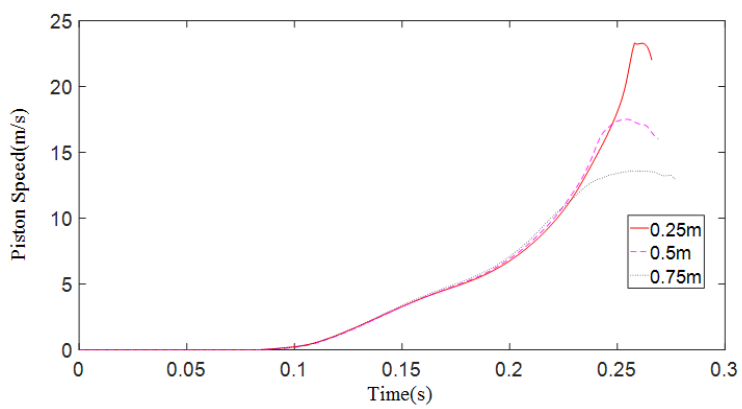

Fig 15 Change of piston speed in different time

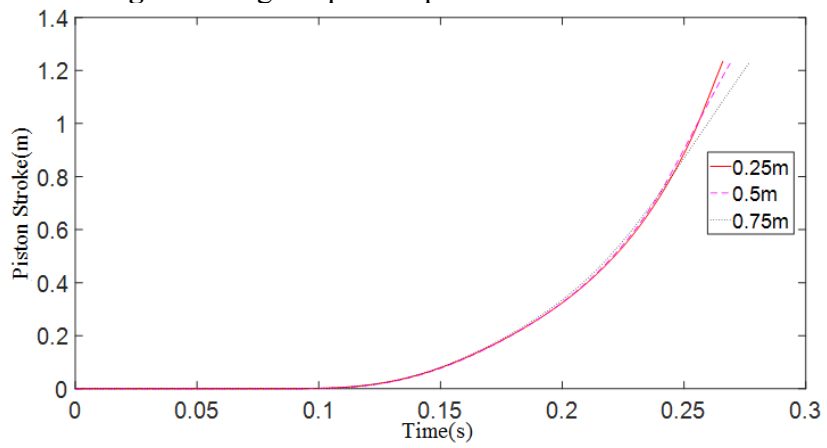

Fig 16 Change of piston stroke in different time

From Fig 14 to Fig 16, it can be found that with the increase of the height of the piston, the acceleration of the piston motion becomes smaller and smaller, and the time required for the same stroke is getting longer and the speed is getting smaller and smaller. This is because the smaller the height of the piston, the greater the mass of the water in the chamber before the outlet, and the more the mass is reduced after the exit, so the acceleration is greater. During the whole course of motion, the final velocity of the three sets of height piston is about $22 \mathrm{~m} / \mathrm{s}, 16 \mathrm{~m} / \mathrm{s}$ and $13 \mathrm{~m} / \mathrm{s}$. The maximum acceleration corresponding to the $0.25 \mathrm{~m}$ piston is about $80 \mathrm{~g}$, the maximum acceleration corresponding to the $0.55 \mathrm{~m}$ piston is about $40 \mathrm{~g}$, the maximum acceleration corresponding to the $0.75 \mathrm{~m}$ piston is about $20 \mathrm{~g}$, and the acceleration corresponding to the $0.25 \mathrm{~m}$ piston is slightly larger. 


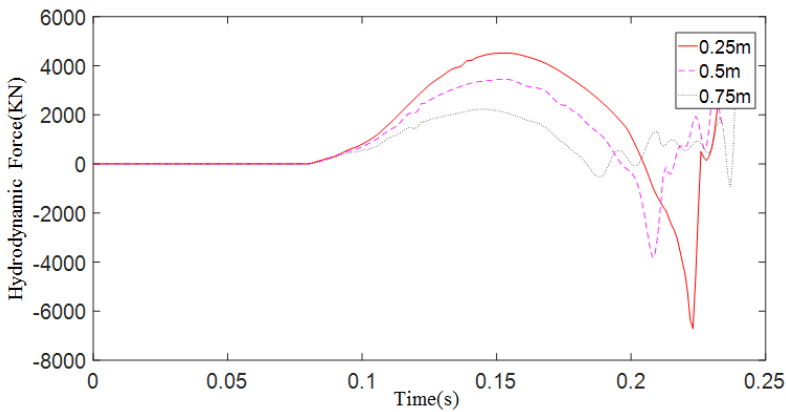

Fig 17 Change of hydrodynamic force in different time

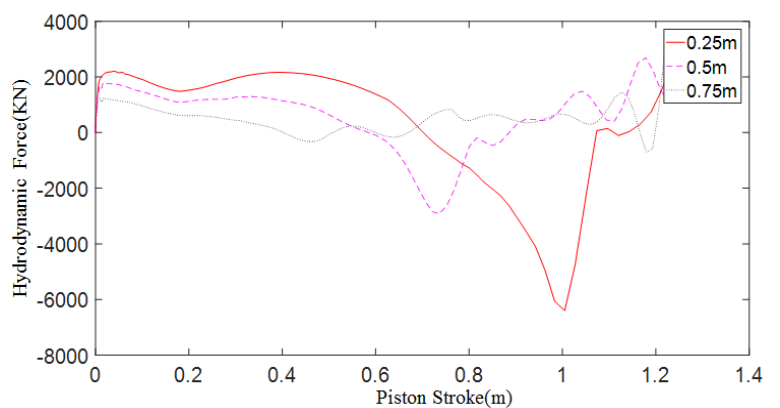

Fig 18 Change of hydrodynamic force in different piston stroke

As seen in Fig 17 and Fig 18, with the increase of the height of the piston, the hydrodynamic force as a whole shows a decreasing trend, and the bottom force does not change much and there is no obvious law of change. This is because the greater the height of the piston, the smaller the velocity, and the positive correlation between the hydrodynamic force and the square of the velocity, so the hydrodynamic force as a whole is decreasing gradually. As seen, curve at $0.75 \mathrm{~m}$ has good performance in hydrodynamic force.

From the curves above, it can be seen that when the initial accumulator pressure was changed, the initial accumulator pressure has little effect on inferior vena pressure, inferior vena force and inferior vena momentum.

Under two kinds of piston-initial accumulator pressure conditions, the variation trend of different piston height is consistent. The product of the three sets of height is not different, and the momentum ratio of the three sets of height is close. The effect of different pressure on piston motion is mainly determined by the pressure boundary of the canister launcher. But when the piston-initial accumulator pressure is reduced, the hydrodynamic force will increase. When the piston height is $0.75 \mathrm{~m}$, the maximum hydrodynamic power will increase from $2100 \mathrm{KN}$ to $2300 \mathrm{KN}$. Therefore, proper increase of piston-initial accumulator pressure is beneficial to reduce hydrodynamic noise.

\section{Conclusions}

Generally some understandings are obtained for designing of the pneumatic underwater launching system. piston.
- The initial accumulator pressure has little effect on inferior vena pressure, inferior vena force and inferior vena momentum.

- Proper increase of piston-initial accumulator pressure is beneficial to reduce hydrodynamic noise. Choose the appropriate pressure of accumulation.

Some simulation work was carried out and the conclusions above were well verified.

\section{References}

1. Y. N. Savchenko, V. N. Semenenko. Unsteady supercavitated motion of bodies. International journal of fluid mechanics research. 2000, 27(1):109 137

2. Chen, Y. and Heister, S.D. Modeling Hydrodynamic Nonequilibrium in Cavitating Flows[J]. Journal of Fluids Engineering. Vol. 118, 1996. pp. 172-178

3. CHENG Y, LIU H. A Coupling Model of Water Flows and Gas Flows in Exhausted Gas Bubbleon Missile Launched Underwater[J].Journal of Hydrodynamics, Ser. B, 2007, 19(4): 403-411

4. Nahra H K, Motil B J, Skor M. Measurements of Shear Lift Force on a Bubble in Channel Flow in Microgravity[M]. National Aeronautics and Space Administration, Glenn Research Center, 2003

5. A.P. Aguiar, J. P. Hespanha. Trajectory-Tracking and Path-Following of Underactuated Autonomous Vehicles with Parametric Modeling Uncertainty. IEEE Transactions on Automatic Control. 2007, 52(8):1362-1379

6. Li J.W., Song B.W., Shao C. Tracking Control of Autonomous Underwater Vehicles with Internal Moving Mass. Acta Automatica Sinica, 2008, 34(10):1319-1323

7. K. Pathak, J. Franch, S. K. Agrawal. Velocity and Position Control of a Wheeled Inverted Pendulum by Partial Feedback Linearization. IEEE Transactions on Robotics. 2005, 21(3): 505-513

8. Zong Zhi. Dong Guohai. A Second Order Volume of Fluid (VOF) Scheme For Numerical Simulation of 2-D Breaking Waves. Journal of Marine Science and Application. 2007, 6(2):1 5

9. Zhou Yiren, Chen Guoping, Wang Dengtin. Experimental study on Total Uplift Force of Waves on Horizontal Plates. Journal of Hydrodynamics, Ser. B. 2004, 16(2): 220 226

10. J. G Graver. Underwater gliders: dynamics, control and design [D]. USA: Princeton University, 2003 